\title{
A comparison of interventional clinical trials in rare versus non-rare diseases: an analysis of ClinicalTrials.gov
}

\author{
Stuart A Bell and Catrin Tudur Smith*
}

\begin{abstract}
Objectives: To provide a comprehensive characterisation of rare disease clinical trials registered in ClinicalTrials.gov, and compare against characteristics of trials in non-rare diseases.

Design: Registry based study of ClinicalTrials.gov registration entries.

Methods: The ClinicalTrials.gov registry comprised 133,128 studies registered to September 27, 2012. By annotating medical subject heading descriptors to condition terms we could identify rare and non-rare disease trials. A total of 24,088 Interventional trials registered after January 1, 2006, conducted in the United States, Canada and/or the European Union were categorised as rare or non-rare. Characteristics of the respective trials were extracted and summarised with comparative statistics calculated where appropriate.
\end{abstract}

Main outcome measures: Characteristics of interventional trials reported in the database categorised by rare and non-rare conditions to allow comparison.

Results: Of the 24,088 trials categorised 2,759 (11.5\%) were classified as rare disease trials and 21,329 (88.5\%) related to non-rare conditions. Despite the limitations of the database we found that rare disease trials differed to non-rare disease trials across all characteristics that we examined. Rare disease trials enrolled fewer participants (median 29 vs. 62), were more likely to be single arm (63.0\% vs. $29.6 \%)$, non-randomised (64.5\% vs. $36.1 \%)$ and open label (78.7\% vs. 52.2\%). A higher proportion of rare disease trials were terminated early (13.7\% vs. 6.3\%) and proportionally fewer rare disease studies were actively pursuing, or waiting to commence, enrolment (15.9\% vs. 38.5\%).

Conclusion: Rare disease interventional trials differ from those in non-rare conditions with notable differences in enrolment, design, blinding and randomisation. However, clinical trials should aim to implement the highest trial design standards possible, regardless of whether diseases are rare or not.

Keywords: Rare disease clinical trial, ClinicalTrials.gov

\section{Background}

In the United States (US), a rare disease is defined as having a prevalence of fewer than 200,000 affected individuals [1]. Across the European Union (EU) the definition is that the condition affects not more than 5 in 10,000 individuals [2]. The Orpha.net database, which provides a reference portal for information on rare diseases, identifies approximately 7,000 rare diseases [3].

It is often assumed that clinical trials in rare conditions differ from those of non-rare conditions. However,

\footnotetext{
* Correspondence: cat1@liverpool.ac.uk

Department of Biostatistics, University of Liverpool, Duncan Building, Daulby Street, Liverpool L69 3GA, UK
}

the extent and nature of these differences is not well understood. Kesselheim et al. provide one comparative survey exploring pivotal trials in orphan versus nonorphan drug approval [4]. These authors characterised a number of preapproval trials highlighting differences in enrolment, randomisation, blinding, comparison groups and primary outcomes. However, their survey was limited to oncology trials that supported successful drug approvals. The approach of Kesselheim et al. was extended by Orfali et al. to clinical trials of non-oncological orphan drugs compared with those of non-orphan drugs [5]. These authors concluded that characteristics such as blinding, randomisation and placebo control were similar 
between trials of orphan vs. non-orphan drugs. However, methodological flaws in their approach likely 'blunted' differences observed between the cohorts [6]. Further studies have explored the pivotal trials and complete dossiers of orphan medicinal products approved by the European Medicines Agency (EMA); although neither publications provided contrasting characteristics for non-orphan products $[7,8]$. To our knowledge no large scale comparative survey has been undertaken to contrast rare disease trials with those in other conditions.

ClinicalTrials.gov provides a public access registry and results database recording clinical studies on human participants. It provides a global registry of both publicly and privately supported trials. The registry is widely used currently containing close to 150,000 research studies. Data can be downloaded across all registered studies and has been used to explore various characteristics of clinical trials $[9,10]$. Recently, the usability of the ClinicalTrials.gov dataset has been extended for research purposes through the development of the Aggregate Analysis of ClinicalTrials. gov (AACT) database [11]. Research using the AACT database to conduct surveys of clinical trials across different clinical specialties is now emerging. Particular examples include Califf et al. who provide specific characterisations of interventional trials in the areas of oncology, cardiovascular and mental health and Hirsch et al. who also characterise interventional oncology trials with further sub-categorisation $[12,13]$.

Our objectives were to use the AACT database to provide a comprehensive characterisation of rare disease clinical trials registered in ClinicalTrials.gov. Further, we wished to compare these characteristics against those of trials in other, non-rare, conditions.

\section{Methods}

\section{The AACT database}

On July 25, 2013 we downloaded the 2012 AACT database from the CTTI website [14]. The database comprised of 133,128 clinical studies registered with ClinicalTrials. gov to September 27, 2012. The key feature of the design, structure and purpose of the AACT database are detailed in Tasneem et al. and the corresponding website $[11,14]$.

When submitting trials to ClinicalTrials.gov data submitters are requested to provide the diseases and clinical conditions under study as Medical Subject Heading (MeSH) terms. Further, ClinicalTrials.gov uses an algorithm provided by the National library of Medicine (NLM) to annotate additional MeSH terms to a given study [11].

The same condition may exist in ClinicalTrials.gov under various synonyms. To help categorise conditions we downloaded the $2013 \mathrm{MeSH}$ thesaurus and the 2013 supplementary concept records $[15,16]$. Each record within these sources contained a MeSH Id, a preferred descriptor name and the names of related concepts which provided synonyms and lexical variations of the preferred descriptor. Table 1 shows an example for Ewing Sarcoma of the information extracted from these sources. In this case 'Sarcoma, Ewing' is the preferred MeSH descriptor and the remaining entries are potential variations all mapped to the same MeSH Id.

For the 133,128 clinical studies in the AACT database a total of 211,063 user submitted conditions had been provided. Of these 144,871 (68.6\%) could be annotated with a MeSH Id. A further 268,796 MeSH terms had been annotated to these trials by the NLM algorithm; these were also matched to their relevant MeSH Id.

\section{Identification of rare diseases}

To subgroup studies into rare and non-rare diseases it remained to identify MeSH Id's related to rare diseases. To identify these we consolidated information from three sources. Firstly, ClinicalTrials.gov identifies trials involving rare diseases within the 'By Topic' display option. This listed a total of 1,430 rare diseases based on the US definition. Secondly, we downloaded documentation entitled 'Rare Disease and Cross References' from OrphanData.org containing 6,767 diseases classified as rare within the EU [17]. As a final resource we extracted rare conditions from the alphabetical lists provided by the Office of Rare Diseases Research [18]. This provided an additional 6,526 rare disease condition names. Upon removing duplicates a total of 11,959 rare disease names, synonyms and lexical variations remained. Using a combination of lookups, pattern matching and manual search a total of 6,389 (54\%) rare disease names, synonyms and lexical variations were matched to their relevant $\mathrm{MeSH}$ Id. In total 4,516 unique MeSH Id's were used and identified as rare. Whilst $54 \%$ may seem a low percentage, many of the 11,959 diseases listed are extremely rare and only recently identified. For quality assessment purposes we performed a Targeted Search of ClinicalTrials.gov using each of the 11,959 disease names as conditions. The total number of studies returned from each search was recorded. For example a targeted search using 'Krabbe disease' as the condition name returned 19 studies that may relate to this disease. In total 194,226 studies were returned via this search method, of which, 177,333 (91\%) where contained within the 6,389 of conditions we identified as having MeSH Id's.

$\begin{aligned} & \text { Table } \mathbf{1} \text { Example of the MeSH database table used to } \\
& \text { identify conditions }\end{aligned}$
\begin{tabular}{lll}
\hline ROW_ID & MESH_TERM & MESH_ID \\
\hline 1 & Sarcoma, Ewing & D 012512 \\
2 & Ewing Sarcoma & D 012512 \\
3 & Ewing's Tumor & D 012512 \\
4 & Sarcoma, Ewing's & D 012512 \\
\hline
\end{tabular}


Downloaded 133,128 trials contained in the 2012

AACT database.

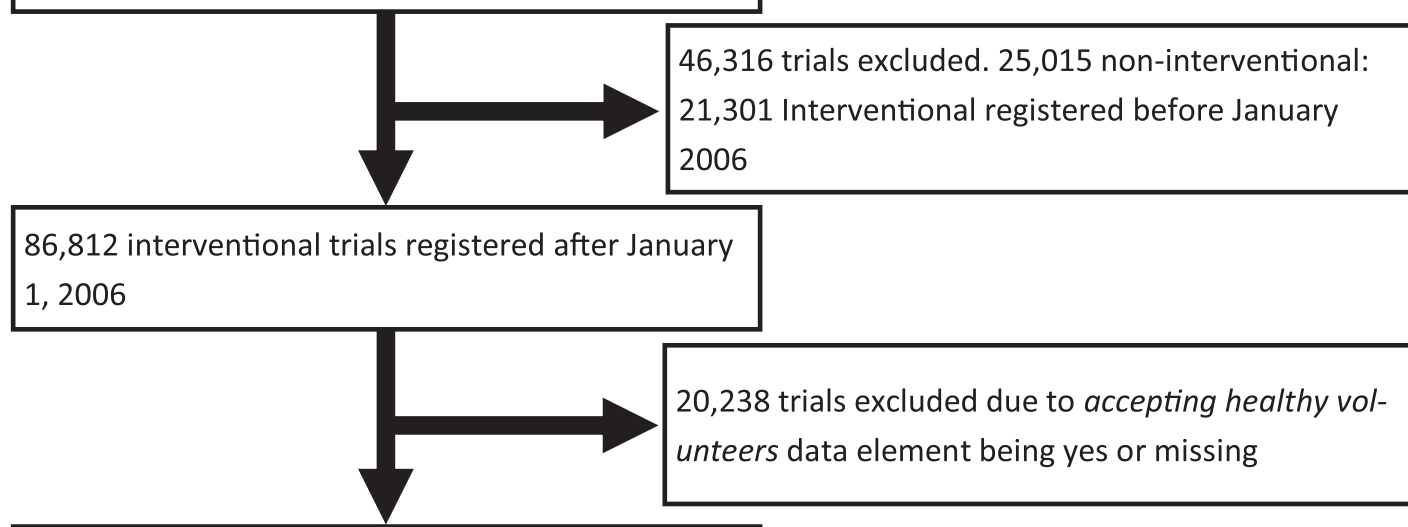

66,574 interventional trials not accepting healthy volunteers

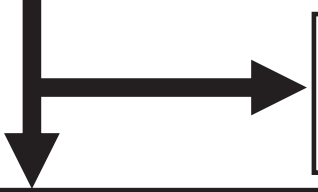

22,588 excluded as location countries either missing or outside US, Canada and EU

Restricted to 43,986 with all location countries in-

side US, Canada and EU

16,020 trials excluded due to missing at least 1

MeSH Id condition annotation

Restricted to 27,966 trials with all conditions

mapped to MeSH Id's.

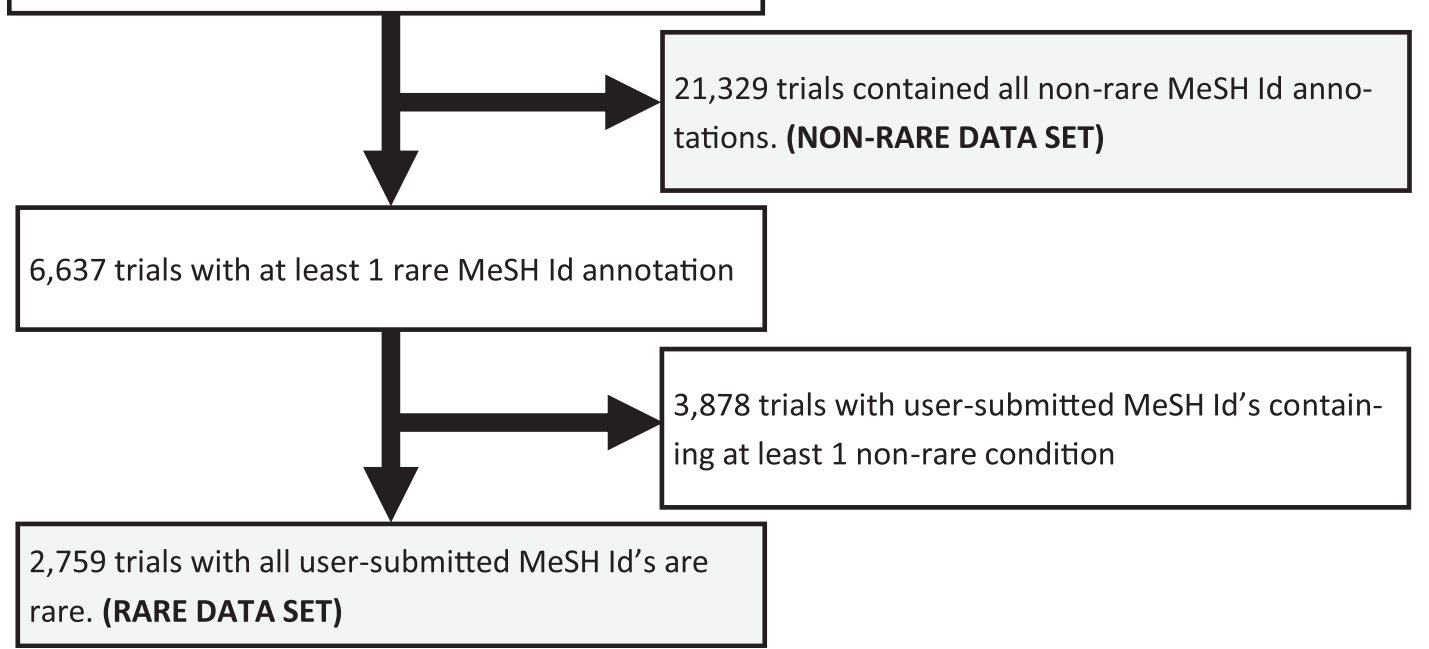

Figure 1 Identification of the comparison data sets (shaded boxes show the final data sets used in analysis. 


\section{Creation of data sets for comparison}

Final datasets for comparison (Figure 1) were created using the annotated MeSH Id's following a number of restrictions. Briefly, of the 133,128 studies in the database only interventional studies were eligible for inclusion leaving 108,113 studies. Attention was restricted to trials first registered on or after January 1, 2006 leaving 86,812 studies.

Our goal was to characterise trials on patients with rare diseases thus any trials accepting healthy volunteers were excluded leaving 66,574 studies. Finally, the conditions identified as rare are only rare in specific countries. We excluded any trial with participating centres registered outside the US, EU or Canada leaving 43,986 studies, of these 27,966 trials had a complete complement of MeSH Id's allowing for categorisation.

A trial was identified as a non-rare disease trial if none of its annotated MeSH Id's had been identified as rare. A total of 21,329 trials had no rare MeSH Id's; 6,637 trials had at least 1 rare disease MeSH Id. Trials were finally classified as rare based on the user submitted MeSH Id's only. We classified a trial as a rare disease trial if all user submitted MeSH Id's were rare; this left 2,759 rare disease trials.

\section{Analytical methods}

Prior to analysis certain missing characteristics were inferred. When a trial relates to a single group with the interventional model described as single assignment the trial was designated as non-randomised with open blinding [12].

Descriptive statistics were used to characterise the identified datasets. For categorical data, frequencies and percentages are stated; for continuous data, medians and interquartile ranges (IQR) are stated. We did not have any pre-specified hypotheses in this study and have not presented results from statistical tests. Between group differences in percentages are presented for categorical characteristics. The $95 \%$ confidence intervals for the between group difference was calculated using the Wilson procedure without continuity correction. R 2.13 was used for all statistical analysis [19].

\section{Ethical approval}

This analysis of existing publicly available data relates to clinical trials and their characteristics rather than human participants. Ethical approval was not required.

\section{Results}

From January 1, 2006 to September 27, 2012 a total of 2,759 trials were identified as relating to rare diseases and 21,329 trials as relating to non-rare diseases. The 2,759 rare disease trials include only 430 unique $\mathrm{MeSH}$ Id's for the conditions under study. This corresponds to only $9.5 \%(430 / 4516)$ of the rare disease unique $\mathrm{MeSH}$
Id's identified. The labels used in the clinicaltrials.gov database are in some sense weakly defined and can often be interpreted differently by different users which might impact the validity of results presented here.

\section{Trial characteristics}

Basic characteristic of trials in the identified datasets are shown in Table 2. The meaning of characteristics is generally self-evident, although exact definitions for certain characteristics are given in the text. Further definitions of characteristics can be found in the ClinicalTrials.gov draft Protocol Data Element Definitions and e-appendix 1 of Califf et al. $[12,20]$.

The proportion of trials which are in rare diseases has decreased over time based on the year enrolment to protocol began (Table 2). The overall recruitment status also suggests a higher proportion of rare disease trials are active but not yet recruiting or have terminated early with fewer currently recruiting.

Study duration is defined as the date from which enrolment begins until the final date on which data was, or is anticipated to be, collected. The median study duration, in years, is longer in rare disease trials (median, 3.2[IQR 2.0-4.9] vs. 2.3[1.3, 3.8]).

Participant eligibility characteristics based on gender and age are more inclusive in rare disease trials with a higher proportion of rare disease trials that included both genders $(93.6 \%$ vs. $86.0 \%)$, paediatric patients $(20.6 \%$ vs. $11.1 \%)$ and elderly patients ( $86.4 \%$ vs. $83.2 \%)$. It should be noted that this is only enrolment criteria and not data on those who actually participated.

In ClinicalTrials.gov a trial can report either actual or expected numbers recruited but not both. The anticipated number of patients to be recruited in rare trials is less than that of non-rare disease trials (median, 41[IQR 24-74] vs. 76[40-182]). Similarly, the actual number of patients recruited is less for rare disease trials than in non-rare trials $(29[12-60]$ vs. $62[27-175])$. For rare disease trials the median actual enrolment is $70.1 \%(29 / 41)$ of the median anticipated enrolment compared to $81.6 \%$ $(62 / 76)$ for non-rare trials and the proportion of trials in rare diseases decreases as enrolment number (anticipated or actual) increases.

The proportion of rare disease trials with an industrial lead sponsor, defined as 'the organization or person who oversees the clinical study and is responsible for analysing the study data'. ClinicalTrials.gov [20], is slightly greater (34.5\% vs. $30.2 \%)$ than non-rare disease trials. The majority of trials of either type are undertaken within a single administrative region (US, Canada or the EU). However, a higher proportion of rare disease trials are undertaken across these administrative regions (8.1\% vs. $4.4 \%)$ compared to non-rare disease trials. Further, exploring individual countries as opposed to these administrative regions, a 
Table 2 Characteristic of rare and non-rare disease clinical trials within the ClinicalTrials.gov registry

\begin{tabular}{cllll}
\hline Characteristics & $\begin{array}{l}\text { Rare disease trials* } \\
(\mathbf{n = 2 7 5 9 )}\end{array}$ & $\begin{array}{l}\text { Non-rare disease trials* } \\
(\mathbf{n = 2 1 3 2 9 )}\end{array}$ & $\begin{array}{l}\text { Difference in percentage points } \\
\text { (rare-non-rare) }\end{array}$ & $\begin{array}{l}\mathbf{9 5 \%} \mathrm{Cl} \text { for } \\
\text { difference }\end{array}$ \\
\hline $\begin{array}{c}\text { Year enrolment to protocol began, } \mathbf{n}(\%) \\
\quad\end{array}$ & $455(16.7)$ & $2791(13.2)$ & 3.4 & 2 to 5 \\
$\quad \begin{array}{l}\text { Prior to } 2006 \\
2006-2007\end{array}$ & $871(31.9)$ & $5121(24.2)$ & 7.6 & 6 to 10 \\
$2008-2009$ & $859(31.5)$ & $5858(27.8)$ & 3.7 & 2 to 6 \\
$2010-2011$ & $467(17.1)$ & $5315(25.2)$ & -8.1 & -7 to -10 \\
2012 and after & $78(2.9)$ & $2018(9.6)$ & -6.7 & -6 to -7
\end{tabular}

Overall status, $\mathrm{n}(\%)$

Completed
Not yet recruiting
Recruiting
Withdrawn
Active, not recruiting
Terminated
Suspended
Enrolling by invitation

Gender, n (\%)

Female
Male
Both

Includes paediatric $(<18), \mathrm{n}(\%)$

Yes

No

Includes Elderly (>65), n (\%)

Yes

No

$1169(42.4)$
$15(0.5)$
$372(13.5)$
$99(3.6)$
$638(23.1)$
$379(13.7)$
$36(1.3)$
$51(1.8)$

$116(4.2)$

$60(2.2)$

$2583(93.6)$

$546(20.6)$

$2106(79.4)$

$2291(86.4)$

$361(13.6)$
$798(61.7)$
280 (21.6)
195 (15.1)

21 (1.6)

$955(71.4)$
$211(15.8)$
$158(11.8)$
$14(1.0)$

$951(34.5)$

72 (2.6)

$15(0.5)$

$1721(62.4)$

$1720(62.3)$

$83(3.0)$
8564 (40.2)

1030 (4.8)

6840 (32.1)

332 (1.6)

2742 (12.9)

$1332(6.2)$

154 (0.7)

335 (1.6)

1949 (9.1)

1042 (4.9)

$18338(86.0)$

2294 (11.1)

$18320(88.9)$

17157 (83.2)

3457 (16.8)

4556 (38.2)

2731 (22.9)

3767 (31.6)

877 (7.4)

3570 (43.3)

1607 (19.5)

2402 (29.1)

$672(8.1)$

6437 (30.2)

$602(2.8)$

$472(2.2)$

$13817(64.8)$

12073 (56.6)

$1387(6.5)$

\section{2}

$-4.3$

$-18.6$

2.0

10.3

7.5

0.6

0.3

$-4.9$

$-2.7$

7.6

9.5

$-9.5$

3.2

$-3.2$

23.5

$-1.3$

$-16.5$

$-5.7$

28.1

$-3.7$

$-17.3$

$-7.1$

4.3

$-0.2$

$-1.7$

$-2.4$

5.7

$-3.5$
0 to 4

-4 to -5

-17 to -20

1 to 3

9 to 12

6 to 9

0 to 1

0 to 1

-4 to -6

-2 to -3

7 to 9

8 to 11

-8 to -11

2 to 5

-2 to -5

21 to 26

0 to -4

-14 to -19

-5 to -6

25 to 31

-1 to -6

-15 to -19

-6 to -8

2 to 6

0.5 to -0.8

-1 to -2

-1 to -4

4 to 8

-3 to -4 
Table 2 Characteristic of rare and non-rare disease clinical trials within the ClinicalTrials.gov registry (Continued)

\begin{tabular}{|c|c|c|c|c|}
\hline EU only & 733 (26.6) & 6938 (32.5) & -6.0 & -4 to -8 \\
\hline US/Canada & $84(3.0)$ & 405 (1.9) & 1.1 & 1 to 2 \\
\hline US/EU & $93(3.4)$ & $333(1.6)$ & 1.8 & 1 to 3 \\
\hline EU/Canada & $9(0.3)$ & $55(0.3)$ & 0 & -0.1 to 0.4 \\
\hline US/EU/Canada & $37(1.3)$ & $138(0.6)$ & 0.7 & 0.3 to 1 \\
\hline \multicolumn{5}{|c|}{$\begin{array}{l}\text { Number of countries, } \\
\text { n (\%) }\end{array}$} \\
\hline Single country & 2442 (88.5) & $19847(93.1)$ & -4.5 & -3 to -6 \\
\hline 2-3 countries & $219(7.9)$ & $1057(5.0)$ & 3.0 & 2 to 5 \\
\hline$\geq 4$ countries & $98(3.6)$ & $425(2.0)$ & 1.6 & 1 to 2 \\
\hline
\end{tabular}

*Denominators exclude missing values. Missing data values [Rare (\%), Non-Rare(\%)] are: Year of enrolment [29(1.1),226(1.1)]; Study duration [361(13.1),2784(13.1)]; Ages [107(3.9),715(3.3)]; Enrolment [127(4.6),1147(5.4)].

¥Anticipated and Actual enrolment are mutually exclusive in ClinicalTrials.gov (Rare Anticipated [1294] vs. Actual [1338]; Non-Rare Anticipated [11931] vs. Actual [8251]).

higher proportion of rare disease trials reported being multi-national ( $11.5 \%$ vs. $6.9 \%)$.

\section{Design characteristics}

Design characteristics of rare diseases trials differ to non-rare diseases trials (Table 3). Centres (labelled as facilities in ClinicalTrials.gov) identify where the protocol is being conducted. A lower proportion of rare disease trials conducted the study protocol at a single centre compared to non-rare trials (61.3\% vs. $72.7 \%$ ).

Rare disease trials are more likely to be early phase trials (those reporting a phase 1 and/or 2 element), (72.5\% vs. $38.5 \%)$. A lower proportion of rare disease trials employed the N/A phase description (11.3\% vs. $30.2 \%)$ which is advised to be used for trials that do not involve drug or biologic products although this is not enforced in ClinicalTrials.gov [20]. The primary purpose of a rare disease trial is generally treatment $(91.2 \%$ vs. $79.3 \%)$ with the intervention used tending to be a drug ( $79.9 \%$ vs. $55.8 \%)$.

The appointment of a Data monitoring committee (DMC) is more common in rare disease trials $(53.2 \%$ vs. $40.9 \%)$. They are also more likely to have a single group assignment $(63.0 \%$ vs. $29.6 \%)$, with no randomisation (64.5\% vs. $28.4 \%)$ and open label ( $78.7 \%$ vs. $52.2 \%)$. Rare disease trials are also more likely to explore both safety and efficacy endpoints in the same trial (63.2\% vs. $45.9 \%)$.

\section{Discussion}

This analysis of ClinicalTrials.gov suggests that trials in rare diseases differ from those of non-rare conditions. Rare disease trials are more likely to have smaller target sample size, more likely to be early-phase, more likely to recruit to a single arm, more likely to be non-randomised, and more likely to be unblinded. These results support those of Kesselheim et al. and Mitsumoto et al. that have explored orphan drug approval trials in the specialities of cancer and neurology respectively $[4,21]$. Results from our study add to current knowledge by including a wider array of clinical studies, not just those completed and used in application for drug approval, and by examining a number of characteristics that have not been considered previously.

It has been argued that whenever possible standard methodological approaches, such as the randomised controlled trial, should be applied in the design and analysis of a clinical trial [22]. However, due to the very limited pool of eligible patients with rare diseases, evidence from good quality randomised controlled trials is often absent for a particular disease. The rarity of the disease makes enrolling a sufficient number of participants inherently difficult.

A higher proportion of rare disease trials employ a multi-centre design; potentially across multiple countries. However, whilst there is an increased use of multi-centre trials in rare diseases compared to non-rare diseases, they are still predominantly single centred. This suggests there is potential for important improvements to be made in the numbers of patients recruited to rare disease trials. Further cooperation between research facilities could greatly improve research and the on-going development of patient contact registries could also serve as powerful tools for improving recruitment [23].

A larger proportion of rare disease trials enrolled paediatric participants particularly in conjunction with adult participants. That $67.9 \%$ of rare disease trials within the orpha.net database had an age of onset given as neonatal, infancy or childhood could go some way to explaining this result. In general, eligibility criteria in rare disease trials were less restrictive than in non-rare trials. Whilst we only have eligibility criteria in terms of age and gender in the ClinicalTrials.gov dataset the idea of the introduction of participant heterogeneity needs to be carefully considered in the analysis plan of any rare disease trials. Failure to identify the most appropriate target population was found to be a key feature of failed orphan marketing authorisations in both the EU and US [24,25]. 
Table 3 Design attributes of clinical trial within the ClinicalTrials.gov data set

Characteristics

$$
\begin{aligned}
& \text { Single facility } \\
& 2-3 \text { facilities } \\
& \geq 4 \text { facilities }
\end{aligned}
$$

Study phase, $\mathbf{n}(\%)$

$\begin{array}{ll}\text { Phase } 0 & 15(0.5) \\ \text { Phase } 1 & 410(14.8) \\ \text { Phase 1/2 } & 294(10.7) \\ \text { Phase } 2 & 1212(43.9) \\ \text { Phase 2/3 } & 83(3.0) \\ \text { Phase 3 } & 287(10.4) \\ \text { Phase 4 } & 145(5.3) \\ \text { NA } & 313(11.3) \\ \text { mary purpose, } \mathbf{n} \text { (\%) } & \\ \text { Treatment } & 2451(91.2) \\ \text { Prevention } & 87(3.2) \\ \text { Screening } & 1(0.0) \\ \text { Supportive Care } & 39(1.5) \\ \text { Health Services Research } & 7(0.3) \\ \text { Educational/Counselling/Training } & 2(0.1) \\ \text { Diagnostic } & 75(2.8) \\ \text { Basic Science } & 25(0.9)\end{array}$

\section{Study has DMC†, $\mathbf{n}(\%)$}

Yes

No

Intervention model¥, $\mathrm{n}$ (\%)

Single Group Assignment
Parallel Assignment
Crossover Assignment
Factorial Assignment

Intervention typeł, $\mathbf{n}(\%)$

Drug

Procedure

Biological

Radiation

Behavioural

Dietary Supplement

Genetic

Other

Allocation $¥ \mathrm{n}$ (\%)

Randomised

Non-randomised

\section{Rare disease trials* Non-rare disease trials*}

( $n=2759$ )

( $n=21329)$

$1671(61.3)$
$333(12.2)$
$720(26.4)$

$15443(72.7)$

$2482(11.7)$

$3315(15.6)$

$166(0.8)$

1848 (8.7)

$1014(4.8)$

4747 (22.3)

601 (2.8)

3110 (14.6)

$3398(15.9)$

6445 (30.2)

$16195(79.3)$
$1488(7.3)$
$86(0.4)$
$844(4.1)$
$446(2.2)$
$14(0.1)$
$917(4.5)$
$441(2.2)$

1211 (53.2)

1066 (46.8)

$7274(40.9)$

10511 (59.1)

$1691(63.0)$
$837(31.2)$
$140(5.2)$
$14(0.5)$

$2204(79.9)$

127 (4.6)

269 (9.7)

$220(8.0)$

112 (4.1)

$47(1.7)$

$31(1.1)$

39 (1.4)

176 (6.4)

949 (35.5)

$1727(64.5)$

$6134(29.6)$

1741 (8.4)

$411(2.0)$

11891 (55.8)

$926(4.3)$

$476(2.2)$

2631 (12.3)

$562(2.6)$

$124(0.6)$

14958 (71.6)
Difference in percentage points (rare-non-rare)

$95 \% \mathrm{Cl}$ for difference

$\begin{array}{ll}-11.4 & -9 \text { to }-13 \\ 0.5 & 0 \text { to } 2 \\ 10.8 & 9 \text { to } 13\end{array}$

$-0.2$

0 to -0.5

6.2

5.9

21.7

0.2

$-4.2$

$-10.7$

$-18.9$

5 to 8

5 to 7

20 to 24

0 to 1

0 to -5

-10 to -12

-17 to -20

12.0

11 to 13

$-4.0$

$-0.4$

$-2.7$

$-2$

0.0

$-1.7$

$-1.2$

-3 to -5

-0.2 to -0.5

-2 to -3

-2 to -2

0 to 0

-1 to -2

-1 to -2

12.3

$-12.3$

10 to 14

-10 to -14

33.5

32 to 35

$-28.9$

$-3.2$

$-1.5$

-27 to -31

0 to -4

24.1

-1 to -2

$2620(12.3)$

22 to 26

$-7.7$

$-1.5$

3.6

1.8

$-10.6$

$-1.5$

0.8

$-6.3$

-7 to -9

0 to -3

3 to 5

1 to 3

-10 to -11

-1 to -2

0 to 1

-5 to -7

-34 to -38

$-36.1$

34 to 38 
Table 3 Design attributes of clinical trial within the ClinicalTrials.gov data set (Continued)

\begin{tabular}{|c|c|c|c|c|}
\hline \multicolumn{5}{|l|}{ Endpoint classification, n (\%) } \\
\hline Efficacy Study & $530(22.0)$ & $7454(42.0)$ & -20.0 & -18 to -22 \\
\hline Safety/Efficacy Study & $1521(63.2)$ & $8148(45.9)$ & 17.3 & 15 to 19 \\
\hline Safety Study & $276(11.5)$ & $1154(6.5)$ & 5.0 & 4 to 6 \\
\hline Pharmacodynamics Study & $9(0.4)$ & $282(1.6)$ & -1.2 & -0.8 to -1.5 \\
\hline Pharmacokinetics Study & $36(1.5)$ & 339 (1.9) & -0.4 & 0.2 to -0.9 \\
\hline Pharmacokinetics/Dynamics Study & $27(1.1)$ & $234(1.3)$ & -0.2 & 0.3 to -0.5 \\
\hline Bio-equivalence Study & $5(0.2)$ & $90(0.5)$ & -0.3 & 0 to -0.5 \\
\hline Bio-availability Study & $1(0.0)$ & $47(0.3)$ & -0.2 & -0.2 to -0.3 \\
\hline \multicolumn{5}{|l|}{ Type of arms $\neq, n$ (\%) } \\
\hline Experimental & $1743(84.3)$ & $13063(73.4)$ & 10.4 & 9 to 12 \\
\hline Active comparator & $458(22.2)$ & $7569(42.8)$ & -20.7 & -19 to -23 \\
\hline Placebo comparator & $343(16.6)$ & $4701(26.6)$ & -10.0 & -8 to -12 \\
\hline Sham comparator & $6(0.3)$ & $346(2.0)$ & -1.7 & -1 to -2 \\
\hline No Intervention & $73(3.5)$ & 1908 (10.8) & -7.3 & -6 to -8 \\
\hline Other & $84(4.1)$ & $111(6.3)$ & 3.4 & 3 to 4 \\
\hline \multicolumn{5}{|l|}{ Blinding, n (\%) } \\
\hline Open & 2137 (78.7) & $10967(52.2)$ & 26.6 & 25 to 28 \\
\hline Single & $89(3.3)$ & $2680(12.8)$ & -9.5 & -9 to -10 \\
\hline Double & $488(18.0)$ & 7370 (35.1) & -17.1 & -15 to -19 \\
\hline
\end{tabular}

Despite these attempts to improve participation rare disease clinical trials are still frequently small, single arm studies. Concerns have been raised over the quality of evidence obtained from such studies [26,27]. It is argued that even in rare disease trials, where we cannot provide a large quantity of evidence, researchers should still attempt to provide good quality evidence [26]. Blinded, randomised controlled trials are classed as the gold standard for clinical trials. Whilst not straight-forward to perform in rare diseases, these standards can still often be achieved. Recent reviews have summarised many alternative trial designs that can provide adequate and well-controlled data for rare disease trials $[28,29]$. Of course, design recommendation for rare disease trials such as Bayesian methods [30] and adaptive randomisation [31] are subtle and not identifiable in ClincalTrials. gov and further analysis of published studies would be required to explore if such recommendations have been implemented.

A higher proportion of rare disease trials explored drug and biological interventions than non-rare diseases trials. One possible explanation is the 1983 Orphan
Drug Act (ODA) in which the term "orphan drug" refers to a drug or biologic. It has been highlighted that the ODA provides a shelter for many biologic treatments as market exclusivity provides a surrogate form of patent. Other legislation, such as the Humanitarian Device Exemption within the Safe Medical Devices Act of 1990, exists to support development of other interventions in rare diseases [32]. Despite these legislative incentives few researchers have explored alternative interventions through clinical trials.

The overall status of rare disease trials shows a proportionately larger number have been terminated, withdrawn or suspended. A comprehensive analysis of why this is the case would be a useful aid for future researchers planning rare disease clinical trials and potentially prevent wasted resources. It is estimated that only $10 \%$ of rare diseases have an available treatment [33]. In fact, in our study we found that only $9.5 \%$ of possible 'rare disease' $\mathrm{MeSH}$ terms identified had an associated clinical trial which corresponds well with this finding. Even for rare diseases with licenced orphan medicinal products, these treatments can still be improved and 
there have been concerns expressed regarding the clinical evidence and lack of efficacy data for these products [8,34]. Joppi et al. reflect that "...nearly all the currently estimated 7,000 rare diseases, with approximately 250 new diseases described annually, still await treatment", as such, the medical need for appropriate clinical research in rare diseases remains high.

\section{Limitations}

Numerous limitations to this study need to be noted. Firstly our categorisation of rare and non-rare trials relied on conditions being submitted as MeSH terms. Whilst this is recommended by ClinicalTrials.gov, it is not enforced. Approximately $30 \%$ of studies were instantly excluded because we could not map conditions to MeSH Id's. Of the 11,959 rare disease terms identified, we could only match $54 \%$ to MeSH Id's. Better application of text matching routines and the use of clinical experts could improve this percentage. A potential consequence is that a number of false negative results may be present in the non-rare disease trial dataset; their correct categorisation could help improve the robustness of this characterisation study. However, taking a random sample of 50 trials from the non-rare dataset we did not find any false-negative results. Thus, we believe that this is unlikely to introduce any bias into the finding. The use of standard vocabulary for conditions submitted to ClinicalTrials.gov could facilitate future research.

Using both US and EU rare disease sources may also limit this study as definitions of rare disease differ in these regions. However, there is likely a large crossover of conditions regardless of regions. Taking a random sample of 50 trials from the rare disease data set found only 4 (8\%) trials that were not listed as rare in both the US and EU. These 4 trials listed the conditions of epithelial ovarian cancer, compartment syndrome, photosensitive epilepsy and focal segmental glomerulosclerosis. They are all listed as rare in the ClinicalTrials.gov registry but prevalence for these conditions was not confirmed in the EU orpha.net database [3].

Califf et al. describe a number of limitations to the ClinicalTrials.gov dataset [12]. Firstly, ClinicalTrials.gov does not register all trials. Registries such as ClinicalTrialsRegister.eu can also be used. Confirmation of temporal trends in the ClinicalTrials.gov dataset may need to be corroborated across registries. This is particularly true for specialist datasets; if large research institute register future studies in a different database this could have large implications on the identification of trends.

Secondly, data elements can be missing or unavailable due to changes in the ClinicalTrials.gov registry definitions, statutory requirements or simply not entered by data submitters. Further to missing data elements, data provided can also be inconsistent or inaccurate. For example a number of trials reported with single arm assignment had also reported a crossover, factorial or parallel intervention model. Furthermore, we did not explore the details behind the labels provided for each trial and we recognise that there may be inaccuracies in definitions such as 'randomized' or 'blinded'. Causes for this may be the expertise of data submitters or a lack of suitable constraints on the data being submitted to ClinicalTrials.gov. Missing data, inconsistencies, and inaccuracies can impact the quality of this, and any other, characterisations of trials. However, we believe that these issues are likely to introduce random error rather than bias since the issues are likely to be distributed similarly across rare and nonrare disease trials.

It is also worth pointing out the strengths of this analysis in that we have provided one of the largest and most complete comparative surveys of rare disease clinical trials to date.

\section{Conclusion}

ClincalTrials.gov and the AACT database can help us characterise clinical research in rare diseases. With the Food and Drug Administration (FDA) and EMA providing significant support for rare disease research it is critical to understand how research is implemented to help identify where potential improvements can be made. The ClinicalTrials.gov dataset shows that trials in rare diseases use fewer participants and have longer duration as might be expected. However, our study also shows that rare disease trials are less likely to use blinding and randomisation than trials in other areas. These are generally regarded as hallmarks of high-quality clinical trial design and this raises concerns over the quality of evidence being supplied by rare disease trials. With higher termination rates for these trials, additional research is required to identify ways to improve the quality, as well as the quantity of rare disease trials.

\section{What this paper adds \\ What is already known on this topic?}

The evaluation of treatments for rare diseases presents a number of challenges for trial practitioners, regulators and policy makers. Within certain clinical specialities rare disease trials have been found to be smaller and to tend to use nonrandomized, unblinded designs compared to trials in non-rare conditions.

\section{What this study adds}

This study contrasts rare and non-rare diseases clinical trials across a broader range of characteristics than previously seen in the literature. Trials in rare and non-rare conditions differ in methodological approach including quantifiable differences in the reported use of randomisation, blinding and data monitoring committees. Rare 
disease trials are smaller, longer and more frequently terminated than those in non-rare conditions.

\section{Availability of supporting data}

Data used for this study are available from the following website http://www.ctti-clinicaltrials.org/what-we-do/analys is-dissemination/state-clinical-trials/aact-database or can be obtained by contacting the corresponding author.

\section{Abbreviations}

AACT: Aggregate Analysis of ClinicalTrials.gov; DMC: Data monitoring committee; FDA: Food and Drug Administration; EMA: European Medicines Agency; EU: European Union; IQR: Interquartile Range; MeSH: Medical Subject Heading; NLM: National library of Medicine; ODA: Orphan Drug Act; US: United States.

\section{Competing interests}

The authors declare that they have no competing interests.

\section{Authors' contributions}

SB had access to all of the study data and takes responsibility for the integrity of the data and accuracy of the data analysis. SB and CTS designed the study. SB acquired the data. SB carried out the statistical analysis with input from CTS. SB and CTS drafted and revised the manuscript. Both authors read and approved the final manuscript.

\section{Funding}

Stuart Bell and Catrin Tudur Smith were supported by Medical Research Council funding from the North West Hub for Trials Methodology Research at the University of Liverpool (G0800792).

Received: 18 July 2014 Accepted: 27 October 2014

Published online: 26 November 2014

\section{References}

1. Office of Rare Diseases Research: Frequently Asked Questions. http://rarediseases.info.nih.gov/about-ordr/pages/31/frequently-askedquestions. (accessed 13 November 2013).

2. The Council of the European Union: REGULATION (EC) No 141/2000. Off J Eur Union 2000, L18/1:5.

3. Orphanet Consortium: Rare disease search. http://www.orpha.net/consor/cgibin/index.php?!ng=EN. (accessed 20 June 2013).

4. Kesselheim AS, Myers JA, Avorn J: Characteristics of clinical trials to support approval of orphan vs nonorphan drugs for cancer. JAMA 2011, 305(22):2320-2326

5. Orfali M, Feldman L, Bhattacharjee V, Harkins P, Kadam S, Lo C, Ravi M, Shringarpure DT, Mardekian J, Cassino C, Cote T: Raising orphans: how clinical development programs of drugs for rare and common diseases are different. Clin Pharmacol Ther 2012, 92(2):262-264. doi:10.1038/ clpt.2012.87 [published Online First: 27 June 2012].

6. Kesselheim AS, Avorn J: Drug labels: a flawed source of data for studying orphan drug approvals. Clin Pharmacol Ther 2012, 92(6):694. doi:10.1038/ clpt.2012.166 [published Online First: 17 October 2012].

7. Picavet E, Cassiman D, Hollak CE, Maertens JA, Simoens S: Clinical evidence for orphan medicinal products-a cause for concern? Orphanet J Rare Dis 2013, 8(1):164. doi:10.1186/1750-1172-8-164 [published Online First: 16 October 2013].

8. Joppi R, Bertele V, Garattini S: Orphan drugs, orphan diseases. The first decade of orphan drug legislation in the EU. Eur J Clin Pharmacol 2013, 69(4):1009-1024. doi:10.1007/s00228-012-1423-2 [published Online First: 23 October 2012].

9. Ross JS, Mulvey GK, Hines EM, Nissen SE, Krumholz HM: Trial publication after registration in ClinicalTrials.Gov: a cross-sectional analysis. PLOS Med 2009, 6(9):e1000144. doi:10.1371/journal.pmed.1000144 [published Online First: 8 September 2009].
10. Subramanian J, Madadi AR, Dandona M, Williams K, Morgensztern D, Govindan R: Review of ongoing clinical trials in Non-small cell lung cancer a status report for 2009 from the ClinicalTrials.gov website. J Thorac Oncol 2010, 5(8):1116-1119. doi:10.1097/JTO.0b013e3181e76159.

11. Tasneem A, Aberle L, Ananth H, Chakraborty S, Chiswell K, McCourt BJ, Pietrobon R: The database for aggregate analysis of ClinicalTrials.gov (AACT) and subsequent regrouping by clinical specialty. PLoS One 2012, 7(3):e33677. doi: 10.1371/journal.pone.0033677 [published Online First: 16 March 2012].

12. Califf RM, Kramer JM, Tasneem A, Zarin DA, Sherman RE, Aberle LH: Characteristics of clinical trials registered in clinicaltrials.gov, 2007-2010. JAMA 2012, 307(17):1838-1847. doi:10.1001/jama.2012.3424.

13. Hirsch BR, Califf RM, Cheng SK, Tasneem A, Horton J, Chiswell K, Schulman KA, Dilts DM, Abernethy AP: Characteristics of oncology clinical trials: insights from a systematic analysis of ClinicalTrials.gov. JAMA Intern Med 2013, 173(11):972-979. doi:10.1001/jamainternmed.2013.627.

14. Clinical Trials Transformation Initiative: AACT Database. http://www.ctticlinicaltrials.org/what-we-do/analysis-dissemination/state-clinical-trials/aactdatabase. (accessed 26 June 2013).

15. US National Library of Medicine: Medical subjects heading. http://www.nlm. nih.gov/mesh/obtain.html. (accessed 26 June 2013).

16. US National Library of Medicine: MeSH record types. www.nlm.nih.gov/mesh/ intro_record_types.html. (accessed 1 July 2013).

17. Orphanet Consortium: Rare diseases and cross-referencing. www.orphadata. org/cgi-bin/inc/product1.inc.php. (accessed 21 June 2013).

18. Office of Rare Diseases Research: Genetic and Rare Diseases Information Center. http://rarediseases.info.nih.gov/gard. (accessed 26/06/2013).

19. R Core Team: R: A Language and Environment for Statistical Computing. Vienna, Austria: R Foundation for Statistical Computing; 2013. http://www.Rproject.org/.

20. ClinicalTrials.gov: Protocol Data Element Definitions (draft). http://prsinfo. clinicaltrials.gov/definitions.html. (accessed 1 July 2013).

21. Mitsumoto J, Dorsey ER, Beck CA, Kieburtz K, Griggs RC: Pivotal studies of orphan drugs approved for neurological diseases. Ann Neurol 2009, 66(2):184-190. doi:10.1002/ana.21676.

22. Gerß W, Köpcke W: Clinical Trials and Rare Diseases. In Rare Diseases Epidemiology. Edited by de la Paz Posada M, Groft SC. Netherlands: Springer; 2010:173-190.

23. Richesson RL, Sutphen R, Shereff D, Krischer JP: The rare diseases clinical research network contact registry update: features and functionality. Contemp Clin Trials 2012, 33(4):647-656. doi:10.1016/j.cct.2012.02.012 [published Online First: 2 March 2012].

24. Putzeist M, Heemstra HE, Garcia JL, Mantel-Teeuwisse AK, Gispen-De Wied CC, Hoes AW, Leufkens HG: Determinants for successful marketing authorisation of orphan medicinal products in the EU. Drug Discov Today 2012, 17(7-8):352-358. doi:10.1016/j.drudis.2011.10.027 [published Online First: 7 November 2011].

25. Heemstra HE, Leufkens HG, Rodgers RP, Xu K, Voordouw BC, Braun MM: Characteristics of orphan drug applications that fail to achieve marketing approval in the USA. Drug Discov Today 2011, 16(1-2):73-80 doi:10.1016/j.drudis.2010.11.006 [published Online First: 20 November 2010].

26. Day S: Evidence-based medicine and rare diseases. Adv Exp Med Biol 2010, 686:41-53. doi:10.1007/978-90-481-9485-8_3.

27. Spodick DH: Randomize the first patient: scientific, ethical, and behavioral bases. Am J Cardiol 1983, 51(5):916-917. doi:10.1016/S0002-9149 (83)80161-1.

28. Cornu C, Kassai B, Fisch R, Chiron C, Alberti C, Guerrini R, Rosati A, Pons G, Tiddens H, Chabaud S, Caudri D, Ballot C, Kurbatova P, Castellan AC, Bajard A, Nony P: Experimental designs for small randomised clinical trials: an algorithm for choice. Orphanet J Rare Dis 2013, 8(1):48. doi:10.1186/17501172-8-48.

29. Gupta S, Faughnan ME, Tomlinson GA, Bayoumi AM: A framework for applying unfamiliar trial designs in studies of rare diseases. J Clin Epidemiol 2011, 64(10):1085-1094. doi:10.1016/j.jclinepi.2010.12.019.

30. Lilford RJ, Thornton JG, Braunholtz D: Clinical trials and rare diseases: a way out of a conundrum. BMJ 1995, 311(7020):1621-1625. doi:10.1136/ bmj.311.7020.1621

31. Chow SC, Chang M: Adaptive design methods in clinical trials - a review. Orphanet J Rare Dis 2008, 3:11. doi:10.1186/1750-1172-3-11.

32. Reaves ND: A model of effective health policy: the 1983 Orphan Drug Act. J Health Soc Pol 2003, 17(4):61-71. doi:10.1300/J045v17n04_04. 
33. Tambuyzer E: Rare diseases, orphan drugs and their regulation: questions and misconceptions. Nat Rev Drug Discov 2010, 9(12):921-929. doi:10.1038/ nrd3275 [published Online First: 9 November 2010].

34. Dupont AG, Van Wilder PB: Access to orphan drugs despite poor quality of clinical evidence. Br J Clin Pharmacol 2011, 71(4):488-496. doi:10.1111/ j.1365-2125.2010.03877.x [published Online First: 11 March 2011].

doi:10.1186/s13023-014-0170-0

Cite this article as: Bell and Tudur Smith: A comparison of interventional

clinical trials in rare versus non-rare diseases: an analysis of

ClinicalTrials.gov. Orphanet Journal of Rare Diseases 2014 9:170.

\section{Submit your next manuscript to BioMed Central} and take full advantage of:

- Convenient online submission

- Thorough peer review

- No space constraints or color figure charges

- Immediate publication on acceptance

- Inclusion in PubMed, CAS, Scopus and Google Scholar

- Research which is freely available for redistribution 